\title{
Síndrome de Tako Tsubo
}

\author{
Pedro Sánchez Llanos ${ }^{a}$, Cristina Sánchez Hernándeza, \\ Rafael García Alonso ${ }^{b}$, Irene del Río Cordovésc
}

\begin{abstract}
a Especialista en Medicina Familiar y Comunitaria. Tutor Colaborador de residentes. Servicio de Atención Rural de Atención Primaria de Villaviciosa de Odón (Madrid).
\end{abstract}

${ }^{\mathrm{b}}$ Especialista en Medicina Preventiva y Salud Pública. Tutor Colaborador de residentes. Servicio de Atención Rural de Atención Primaria de Villaviciosa de Odón (Madrid).

c Especialista en Medicina Familiar y Comunitaria. Servicio de Atención Rural de Atención Primaria de Villaviciosa de Odón (Madrid).

Correspondencia: Pedro Sánchez Llanos, Avenida de Italia n 146, 28939 Arroyomolinos (Madrid). Telf.: 686656139, e-mail: pedrinicilina@hotmail.com.

Recibido el 13 de marzo de 2010 .

Aceptado para su publicación el 16 de mayo de 2010.

\begin{abstract}
RESUMEN
El síndrome de Tako Tsubo (STS) o síndrome de disquinesia apical transitoria (SDAT) se describió por primera vez en la década de los noventa en Japón ${ }^{1}$. Es una entidad poco frecuente cercana al $1 \%$ de todos los pacientes con sospecha de síndrome coronario agudo $(\mathrm{SCA})^{2}$. Generalmente afecta a mujeres postmenopaúsicas con pocos factores de riesgo cardiovascular (FRCV) ${ }^{3}$. Se caracteriza por dolor precordial anginoso, cambios electrocardiográficos, elevación de las enzimas de daño miocárdico ${ }^{2}$, ausencia de obstrucción coronaria en la angiografía y una característica disquinesia antero apical del ventrículo izquierdo que se normaliza en unos pocos días ${ }^{4}$. El estrés emocional severo es el desencadenante más común ${ }^{5}$. La etiopatogénesis de este síndrome está aún por definir ${ }^{1}$.

Palabras clave. Cardiomiopatía de Takotsubo, Disfunción Ventricular.
\end{abstract}

\section{ABSTRACT}

\section{Tako Tsubo's syndrome}

The Tako Tsubo syndrome or transient apical dyskinesia syndrome was first described in Japan in the $1990 \mathrm{~s}^{1}$. Is a rare condition $1 \%$ of all the patients with suspicion of acute coronary syndrome ${ }^{2}$. It generally appears in post menopausal women with few cardiovascular risk factors ${ }^{3}$. It is characterized by anginal chest pain, electrocardiographic changes, elevation of the enzymes of myocardial injury ${ }^{2}$, no coronary obstruction on angiography and a characteristic left ventricular antero apical dyskinesia that recovers to normal in a few days ${ }^{4}$. Severe emotional stress is the most common trigger for this syndrome ${ }^{5}$. The aethiopatogenesis of this syndrome has yet to be defined ${ }^{1}$.

Key words. Takotsubo Cardiomyopathy, Ventricular Dysfunction.

\section{INTRODUCCIÓN}

El dolor torácico en atención primaria supone un desafío clínico importante. En el $20 \%$ de los pacientes que acuden por este motivo existe una causa cardiaca $^{6}$. El objetivo es un correcto diagnóstico diferencial de las situaciones potencialmente graves para la inmediata toma de decisiones.

\section{OBSERVACIONES CLÍNICAS}

Presentamos el caso de una mujer de 69 años que mientras se duchaba sufrió quemadura por escaldadura en región glútea izquierda. Con la intensificación del dolor por la quemadura se inició dolor centrotorácico opresivo irradiado al brazo izquierdo con cortejo vagal y sin disnea, persistiendo a su llegada al centro.

Entre los antecedentes personales cabe destacar hipercolesterolemia en tratamiento con simvastatina, hipertensión sin tratamiento y obesidad grado 1 .

En la exploración física encontramos: TA MSD 160/90 mmhg y MSI 155/95 mmhg, FC $90 \mathrm{lpm}$, Sat. O2 98\%, no signos de distrés respiratorio, cortejo vagal, lesión eritematosa en glúteo izquierdo con gran flictena de contenido 
líquido claro, no ingurgitación yugular, auscultación cardiaca y pulmonar normal. El resto de la exploración fue anodina, salvo signos de insuficiencia venosa crónica.

Se realizó ECG de 12 derivaciones con dolor: ritmo sinusal a $75 \mathrm{lpm}$, con PR, QRS y QTc normales, eje QRS izquierdo sin llegar a completar criterios de HBARIHH y no alteraciones agudas de la repolarización.

Se administró nitroglicerina sublingual, disminuyendo el dolor, aunque no llegó a remitir. Repetimos el ECG con menos dolor, pero no se objetivó cambio alguno. Ante la sospecha de dolor torácico de origen coronario, se procedió a medidas de soporte, antiagregación y traslado hospitalario en UVI móvil.

En el hospital persistió leve dolor. No presentó cambios en el ECG. Se realizaron determinaciones analíticas incluyendo hemograma, estudio de coagulación y bioquímica, resultando normales, exceptuando leve elevación de mioglobina de $70 \mathrm{ng} / \mathrm{ml}$ (valores normales: 19-51) y de troponina I (Tnlc) de $0.90 \mu \mathrm{g} / \mathrm{L}$ (valores normales: 0-0.2), con CPK normal. La radiografía de tórax no presentó alteraciones significativas.

La paciente ingresó en $\mathrm{UCl}$, mostrando en el ecocardiograma realizado una aquí-disquinesia de segmentos medio apicales con hipercontractilidad de segmentos basales. Se realizó coronariografía precoz, donde se objetivó un ventrículo izquierdo
(VI) no dilatado con hipoquinesia moderada de segmento medio anterolateral, restos de segmentos con contractilidad conservada y FEVI conservada.

Durante su estancia en $\mathrm{UCl}$ se inició tratamiento antiagregante, anti Ilbllla, HBPM, beta bloqueante e IECAS, permaneciendo asintomática. El ecocardiograma transtorácico al alta confirmó mejoría de la alteración de la contractilidad y VI normal con FEVI conservada. Se interpretó como miocardiopatía por estrés "apical ballooning" o STS.

\section{COMENTARIOS}

En 1990 se describió en Japón un nuevo síndrome caracterizado por una extensa acinesia con abombamiento apical del VI sin lesiones coronarias. Estos pacientes presentan dolor torácico, alteraciones isquémicas agudas en el electrocardiograma (ECG) y una discreta elevación de enzimas de daño miocárdico, similar a pacientes con IAM u otras formas de SCA. Debido a la forma adoptada por el VI en la angiografía en telesístole se acuño el término de STS, ya que recuerda al contorno de una vasija usada en Japón para atrapar pulpos llamada de la misma forma (figura1). El pronóstico es benigno y las alteraciones segmentarias de la contractilidad se recuperan en pocas semanas ${ }^{7}$, por ello recibe la denominación de SDAT. Hasta en el $75 \%$ de los casos se precede de un factor estresante emocional o físico $^{6}$ (en el caso presentado fue tras una quemadura). El factor emocional más descrito suele ser el fallecimiento de una persona allegada ${ }^{8}$. La mayoría de los casos afecta a mujeres postmenopaúsicas

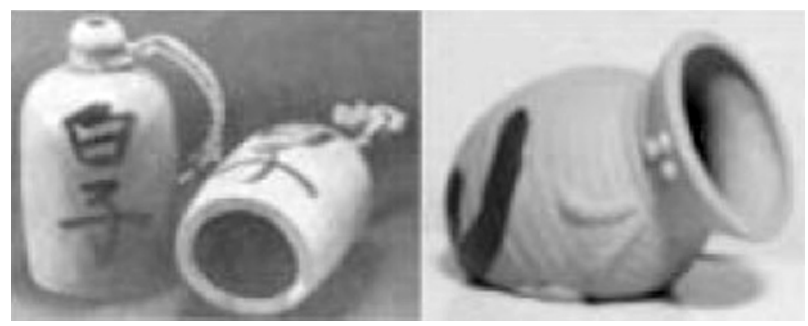

Figura 1. Vasija tradicional japonesa de la que toma el nombre el síndrome de Tako Tsubo.

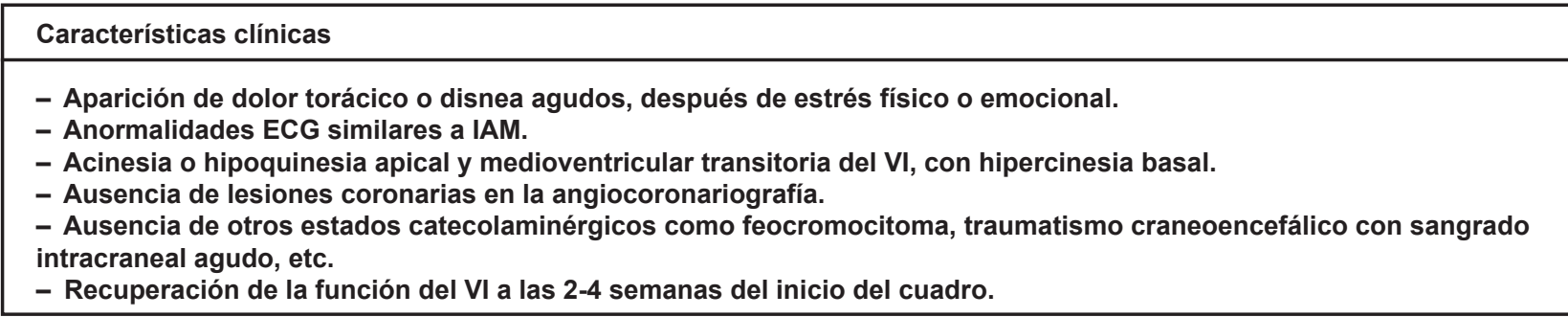

Tabla 1. Características clínicas del Síndrome de Tako-Tsubo. ECG: Electrocardiograma, IAM: Infarto agudo de miocardio, VI: Ventrículo izquierdo. 
Criterios de la Clínica Mayo

1. Discinesia o acinesia transitoria de los segmentos apicales y medios del VI, con anormalidades en la motilidad regional de la pared (de distribución vascular correspondiente a más de una arteria epicárdica).

2. Ausencia de enfermedad coronaria obstructiva o evidencia de rotura aguda de placa en la angiografía.

3. Nueva aparición de anomalías en el ECG (como elevación del segmento ST o inversión de la onda T).

4. Ausencia de feocromocitoma o miocarditis.

Tabla 2. Criterios de la Clínica Mayo para el diagnóstico del STS. VI: Ventrículo izquierdo, ECG: Electrocardiograma.

con pocos $\mathrm{FRCV}^{9}$. La estimación de STS se sitúa alrededor del $1 \%$ del total de IAM $^{10}$. En mujeres es cercano al $5 \%$.

Existe acuerdo general en considerar el STS como una forma de aturdimiento miocárdico, pero su etiopatogenia aún es desconocida ${ }^{7}$. Los mecanismos más frecuentemente invocados son: daño miocárdico directo por catecolaminas ${ }^{11}$, precedido por un factor estresante (por ello también se denomina miocardiopatía por estrés), obstrucción dinámica aguda, severa y transitoria del tracto de salida del VI en pacientes predispuestos (septo sigmoide y tracto estrecho), espasmo coronario localizado o multivaso, alteraciones de la microcirculación y miocarditis y variantes anatómicas predisponentes de la coronaria descendente anterior ${ }^{12}$.

Clínicamente el solapamiento de síntomas y hallazgos iniciales hace difícil distinguir el STS de un SCA. Las características distintivas de STS quedan reflejadas en la tabla 1 . Aunque no hay todavía un claro consenso respecto a los criterios que definen el STS, el modelo diagnóstico más comúnmente aceptado es el propuesto por Bybee (Clínica Mayo), con leves modificaciones sugeridas por Prasad et al (tabla 2$)^{6}$.

El dolor torácico similar al del SCA aparece en un $53-71 \%$ y la disnea en el $7-20 \%$. Como complicaciones pueden aparecer arritmias ventriculares en el $9 \%$, bradicardia severa en el $10 \%$, algún grado de ICC en el $22 \%$ y shock cardiogénico en el $5 \% 4$. La mortalidad se estima entre un 1 y un $3 \%$ en distintas series ${ }^{9}$. La recuperación clínica y mejoría hemodinámica comienza al $2^{\circ}-3^{\circ}$ día, pero los cambios en el ECG pueden durar días o semanas (3-31dias) $)^{4}$. Las recurrencias ocurren en menos del $10 \%$ de pacientes ${ }^{13}$.

EI ECG inicial puede ser similar al de un SCA con elevación del ST en precordiales (90\%), onda T negativa en precordiales (44\%) y onda Q $(15-27 \%)$, siendo rara la imagen especular en cara inferior. No debemos olvidar que el ECG puede ser normal ${ }^{9}$, como en el caso presentado. En cuanto a las enzimas cardiacas, pueden elevarse levemente la CPK en el $56 \%$ y la Troponina casi en el $100 \%$ de los casos, con una curva que evoluciona rápidamente a la normalización. La ecocardiografía realizada inicialmente muestra una disquinesia o aquinesia del ápex del VI, con normo o hipoquinesia basal ${ }^{4}$. La repetición tras la fase aguda confirmará que las anomalías han revertido?.

La coronariografía no muestra lesiones significativas coronarias. En la ventriculografía se objetiva una disfunción del VI con hipocinesia, acinesia o discinesia de los segmentos apicales con hipercontractilidad de los basales, dando como resultado una balonización del VI durante la sístole ${ }^{1}$.

En cuanto al tratamiento no se dispone de datos a partir de ensayos clínicos ${ }^{6}$. Dado que inicialmente a veces es indistinguible del SCA, el tratamiento inicial debería ser el de la isquemia coronaria, con monitorización, oxigenoterapia, AAS, inhibidores de la glicoproteina Ilb/llla y/o anticoagulantes con inhibición directa de la trombina, nitratos, beta bloqueantes, IECA y diuréticos si lo precisa la situación clínica ${ }^{9}$.

En definitiva, hemos presentado un caso de dolor torácico prolongado tras una quemadura (factor estresante), en una mujer con algún FRCV, con ECG realizados con dolor sin signos de isquemia, CPK seriadas normales y mínima elevación de la troponina, coronarias sin lesiones angiográficas, defectos transitorios de la contractilidad segmentaría en el ecocardiograma y coronariografía sugestiva de STS. Salvo la normalidad ECG (también descrita en la literatura), el cuadro es típico de este síndrome, que deberemos sospechar en consulta ante un dolor torácico en una mujer postmenopaúsica con pocos o ningún FRCV precedido de un factor estresante físico o emocional.

\section{BIBLIOGRAFÍA}

1. Katscher W, Casal Sánchez AJ, Sanmartín Férnández M. Síndrome Tako-Tsubo simulando infarto agudo de miocardio. Emergencias. 2006; 18:309-11.

2. Moreno Castillón $C$, Torre Solé N, Sulé Salvado M.A, Plana Blanco A, Marí López A, Bartolomé Matéu S. El gran imitador del infarto agudo de miocardio. Semergen. 2009; 35(9):469-71.

3. Gianini M, Dentali F, Grande AM. Apical Ballooning syndrome of Takotsubo cardiomyopathy: a systematic review. Eur Heart J. 2006; 27(13):1523-9.

4. Salaverría Garzón I, Villaseñor Navas M, Sánchez Herrera S, Martínez Elbal L. Síndrome de Tako-Tsubo (discinesia apical transitoria): Un síndrome que simula un 
infarto de miocardio. An Med Interna (Madrid) [revista en la Internet]. 2008; 25(1):20-2. [citado el 15/02/2010]. Disponible en: http://scielo.isciii.es/scielo.php?script=sci_ arttext\&pid=S0212-71992008000100005\&Ing=es.

5. Buchholz S, Rudan G. Tako-tsubo syndrome on the rise: a review of the current literature. Postgrad Med J. 2007; 83:261-4.

6. Montroig Rodríguez A, Mitjavila López J, Fornés Ollé B. Cardiomiopatía de Tako-Tsubo: una causa peculiar de dolor torácico agudo en atención primaría. FMC. 2009; 16(8):528-33.

7. Ibáñez B, Navarro F, Farré J, Marcos-Alberca P, Orejas $M$, Rábago $R$ et al. Asociación del síndrome de TakoTsubo con la artería coronaría descendente anterior con extensa distribución por el segmento diafragmático. Rev Esp Cardiol. 2004; 57:209-16.

8. Metzl MD, Altman EJ, Spevack DM, Doddamani S, Travin MI, Ostfeld RJ. A case of Takotsubo cardiomyopathy mimicking an acute coronary syndrome. Nat Clin Pract Cardiovasc Med. 2006; 3:53-6.
9. Derrick D. The"broken heart syndrome": understanding Takotsubo cardiomyopathy. Crit Care Nurse. 2009; 29(1):49-57.

10. Previtali M, Repetto A, Panigada S, Camporotondo R, Tavazzi L. Left ventricular apicall ballooning syndrome: Prevalence, clinical characteristics and pathogenetic mechanisms in a European population. Int $\mathrm{J}$ Cardiol. 2008; 134(1):91-6.

11. Abe $\mathrm{Y}$, Kondo M, Matsuoka $\mathrm{R}$, Araki M, Dohyama K, Tanio $\mathrm{H}$. Assesment of clinical features in transient left ventricular apical ballooning. J Am Coll Cardiol. 2003; 41:737-42.

12. Moro JA, Arnau MA, Sánchez E, Almenar L. Síndrome takotsubo con lesiones coronarias acompañantes. Rev Esp Cardiol. 2006; 59:632-3.

13. Prasad A, Lerman A, Rihal CS. Apical ballooning syndrome (Tako-tsubo or stress cardiomyopathy): a mimic of acute myocardial infarction. Am Heart J. 2008; 155(3):408-17. 